\title{
Diversidad de género en posiciones estratégicas y el nivel de endeudamiento: evidencia en empresas cotizadas mexicanas ${ }^{1}$
}

\author{
Diana Denisse Mendoza Quintero \\ Universidad Autónoma de San Luis Potosí \\ Guadalupe del Carmen Briano Turrent ${ }^{2}$ \\ Universidad Autónoma de San Luis Potosí \\ María Luisa Saavedra García \\ Universidad Nacional Autónoma de México
}

(Recepción: 30/mayo/2018, aceptado: 7/septiembre/2018)

\section{Resumen}

El objetivo de este estudio es analizar el efecto de la diversidad de género en el consejo de administración y equipo directivo sobre el desempeño financiero en 98 empresas que cotizan en la Bolsa Mexicana de Valores, durante el periodo 2004-2016. Para ello se adopta un panel de datos no balanceado con efectos fijos y un análisis de regresión Logit para sustentar el análisis econométrico. Los resultados evidencian que a pesar de una escasa participación de mujeres en posiciones estratégicas, éstas tienen un efecto negativo en el nivel de endeudamiento, especialmente cuando se integran al consejo de administración. En relación a la rentabilidad no se evidencia una influencia significativa. Es necesario promover la diversidad de género en posiciones de liderazgo a través de la generación de políticas de inclusión y planes de capacitación y promoción. Si bien el estudio se limita a empresas cotizadas en México, es pionero en analizar la influencia que tiene la mujer en las decisiones financieras, los resultados son un referente para los responsables de emitir las políticas y principios de gobierno corporativo en México.

Clasificación JEL: G32, G34

Palabras clave: Diversidad de Género, Endeudamiento, Equipo Directivo, Teoría de Agencia, México

\section{Gender Diversity on Strategic Positions and the Level of Indebtedness: Evidence from Mexican Listed Companies}

\section{Abstract}

The purpose of this study is to analyze the effect on financial performance of gender diversity in the board of directors and the management team of 98 companies listed in the Mexican Stock Exchange during the period of 2004 to 2016. To this end, unbalanced panel data with fixed effects and a Logit regression analysis are adopted to support the econometric analysis. The results show that despite the low participation of women in strategic positions, they have a negative effect on the level of indebtedness, especially when integrated into the board of directors. With respect to profitability, there is no evidence of significant influence. It is necessary to promote gender diversity in leadership positions through the generation of inclusion policies and training and promotion plans. Although the study is limited to companies listed in Mexico, it does pioneer in analyzing the influence of women on financial decisions, and the results serve as a reference for those responsible of issuing corporate governance policies and principles in Mexico.

\footnotetext{
${ }^{1}$ Agradecimientos. Agradecemos al Consejo Nacional de Ciencia y Tecnología (CONACYT) por el apoyo financiero proporcionado a través del proyecto de Ciencia Básica CB-257211-201.

${ }^{2}$ Sierra Leona No. 550. Lomas 2a. Sección. San Luis Potosí, S.L.P., México. Correo electrónico: guadalupe.briano@uaslp.mx
} 
632 REMEF (The Mexican Journal of Economics and Finance)

Diversidad de género en posiciones estratégicas y el nivel de endeudamiento: Evidencia en empresas cotizadas mexicanas

\section{Abstract}

JEL Classification: G32, G34

Keywords: Gender Diversity, Indebtedness, Management Team, Agency Theory, Mexico

\section{Introducción}

La diversidad de género ha tomado gran relevancia en el campo del gobierno corporativo y de las finanzas corporativas a nivel global. Diversos países, principalmente en Europa, han impuesto cuotas de participación femenina en el consejo de administración, mientras que otras economías han adoptado recomendaciones de aplicación voluntaria para promover una mayor diversidad de género en posiciones estratégicas de la empresa (Chapple Humphrey, 2014). A pesar de que la participación de las mujeres en el mercado laboral ha ido ganando más terreno, a nivel directivo su presencia e influencia en el desempeño empresarial es limitado, particularmente en el contexto de México. De acuerdo Graham, Belliveau y Hotchkiss (2017) el mayor obstáculo que las mujeres enfrentan para ocupar cargos de liderazgo se refiere a mayores responsabilidades familiares en comparación con los hombres.

El comportamiento entre hombres y mujeres ante la toma de decisiones financieras se ha abordado desde las perspectivas de la psicología cognitiva y conductual, encontrando relación con factores como el procesamiento de la información, la diligencia, el conservadurismo, el exceso de confianza y la tolerancia al riesgo (Costa et al. 2001; Eckel Grossman 2002; Nettle 2007; Croson Gneezy 2009). Son diversos los estudios que concluyen que la diversidad de género en el consejo y/o posiciones directivas mejora el desempeño financiero y económico en los países (Australian Securities Exchange, 2010; More, 2017). En la misma línea, Rodríguez Nava y Venegas Martínez (2012) afirman que la diversidad de género conduce a resultados empresariales más sólidos y presentan un comportamiento corporativo para asumir menos riesgos. Faccio, Marchica y Mura (2016) afirman que aquellas empresas en donde una mujer ocupa la posición de director general (CEO), cuentan con niveles de endeudamiento más bajos, ganancias menos fluctuantes y por ende cuentan con mayores posibilidades de supervivencia en comparación con aquellas lidereadas por hombres. Los resultados no son concluyentes y varían de acuerdo al contexto y las preferencias de los tomadores de decisiones. De acuerdo con Khaw et al. (2016), es necesario estudiar el impacto de la diversidad de género en el desempeño financiero de países emergentes como México.

De acuerdo con Adams y Ferreira (2009), la presencia de mujeres en el consejo motiva a una mayor función supervisora en el equipo directivo, lo cual favorece el desempeño corporativo en contextos con un sistema institucional débil y sistemas de gobierno corporativo poco desarrollados (Nguyen, Locke, Reddy, 2015). La presencia femenina en el consejo ha sido asociada a la toma de riesgos y al estilo de liderazgo, lo cual incide directamente en el resultado financiero. Para la teoría de agencia, la independencia del consejo fortalece el entorno institucional en países con una baja protección del accionista minoritario, al contrarrestar la discrecionalidad de los grupos mayoritarios (Pindado De la Torre, 2006). Existe además una relación efectiva entre esa participación femenina y el nivel de deuda, lo que puede explicarse desde la creencia, compartida en otros países, de que la diversidad es un detonante fundamental de la independencia del consejo y de que son precisamente los consejeros independientes los que logran la reputación y credibilidad necesaria para acceder más fácilmente al financiamiento. Por su parte, otros estudios 
afirman que las mujeres adoptan un comportamiento de aversión al riesgo en la toma de decisiones, lo cual podría incidir negativamente en los niveles de endeudamiento y desempeño financiero (Srinidhi et al., 2011). Estudios realizados para empresas cotizadas en México, como el de Camarena, Saavedra y Aguilar (2016), encontraron que el rendimiento y apalancamiento financiero de las empresas que no cuentan con la participación de mujeres en el consejo de administración es mayor, evidenciando la aversión de las mujeres para asumir riesgos, variable que se encuentra ligada a la rentabilidad y que estaría explicando también el menor rendimiento.

El presente trabajo tiene como objetivo analizar la influencia de la participación femenina en el consejo de administración y equipo directivo sobre el nivel de endeudamiento en empresas cotizadas de la Bolsa Mexicana de Valores durante el periodo 2004-2016. México se caracteriza por un entorno institucional débil y un sistema de gobierno corporativo que no ha incluido en sus prácticas el tema de diversidad de género, por lo que el presente trabajo pretende sensibilizar de la importancia de la diversidad de género en la cúspide organizacional, en donde se presentan mayores obstáculos para que las mujeres accedan a cargos directivos o como consejeras de empresas. Esta investigación contribuye en cuatro dimensiones importantes a la literatura y el campo profesional en el ámbito de las finanzas. En primer lugar, extiende la literatura internacional en diversidad de género en el consejo de administración y equipo directivo, lo cual difiere de estudios previos que generalmente se han centrado solamente en el consejo de administración. En segundo lugar, muestra el empleo de modelos econométricos robustos en una muestra amplia de empresas cotizadas mexicanas atendiendo los problemas de multicolinealidad, heterocedasticidad y correlación entre las variables. En tercer lugar, se adoptan las teorías de agencia y de la masa crítica para sustentar las hipótesis de estudio y resultados obtenidos. Este estudio es uno de los pioneros en México en analizar la relación entre la diversidad de género y las decisiones de endeudamiento en empresas cotizadas que incluye una muestra amplia de 874 observaciones año. En cuarto lugar, los resultados de esta investigación podrían ser un referente para la generación de políticas y prácticas de gobierno corporativo que motiven a incrementar la diversidad de género en posiciones estratégicas del sector privado en México.

Este trabajo se divide en cinco secciones. La primera, describe el marco contextual de la diversidad de género en México. Posteriormente se sustentan las hipótesis de estudio. La tercera sección, describe la metodología de investigación empleada. La cuarta sección, presenta la discusión de los resultados descriptivos y econométricos. La última sección, concluye e identifica las limitaciones y líneas futuras de investigación.

\section{Marco Teórico}

\subsection{Diversidad de género en Latinoamérica y México}

La diversidad de género puede ser definida como la presencia de por lo menos una mujer en posiciones de liderazgo, y es una variable que ha sido ampliamente utilizada para estudiar su influencia en diferentes variables financieras (Adams Ferreira, 2009; Campbell Minguez-Vera, 2008). En este sentido, Esteban (2007) refiere que la diversidad de género en el Consejo de Administración, es una muestra de Responsabilidad Social Corporativa, fomenta la creatividad en la toma de decisiones, representa la realidad corporativa, aumenta la responsabilidad de la empresa, entre otros.

Un estudio realizado por el Grant Thornton en 2017, indica que durante 2015 y 2016 el $9 \%$ de las posiciones de director general (CEO) a nivel mundial estaba representado por mujeres, mientras que para 2017 este porcentaje presentó un incremento de 3 puntos porcentuales, siendo aún bajo comparado con los cargos ocupados por los hombres. Con 
634 REMEF (The Mexican Journal of Economics and Finance)

Diversidad de género en posiciones estratégicas y el nivel de endeudamiento: Evidencia en empresas cotizadas mexicanas

respecto a las tasas de participación femenina en el mercado laboral y en especial en el equipo directivo, el porcentaje de mujeres en posiciones de liderazgo representó el $25 \%$ en 2016, y éste se incrementó solamente en un 1\% durante el 2017 (Grant Thornton, 2017). En México, el $36.6 \%$ del empleo formal y el $43.9 \%$ de la población económicamente activa (PEA) estaban representados por mujeres (INEGI, 2017). Al respecto, la Organización para la Cooperación y el Desarrollo Económicos (OCDE), sostiene que pese al crecimiento de la participación femenina en el mercado laboral, las mujeres tienden a ocupar puestos de trabajo con menores salarios y esquemas laborales muy rígidos (El Economista, 2017). De acuerdo a cifras de la OCDE, la inclusión de mujeres en puestos directivos de las empresas ha avanzado de $12 \%$ en 2010 a $20 \%$ en 2016. Sin embargo, en la mayoría de los países que integran la OCDE, las mujeres ocupan menos del $25 \%$ de los lugares en los consejos de administración de las empresas que cotizan en Bolsa. En México, esta proporción es tan solo de un $5 \%$, lo que nos ubica muy por debajo del promedio de los países de la OCDE y por debajo de otros países como Colombia y Brasil, en donde las mujeres ocupan cerca del $11 \%$ y $6 \%$ de estas posiciones (OCDE, 2016). De acuerdo al estudio realizado por ONU Mujeres en México (2016), el porcentaje de mujeres en puestos de liderazgo y con responsabilidades directivas es de $29 \%$ y su presencia en puestos intermedios de las grandes corporaciones del país no supera el $23 \%$. En posiciones directivas, el porcentaje de mujeres se reduce a $13 \%$ y tan solo $5 \%$ son presidentes de consejos directivos. Además la brecha salarial de las mujeres con respecto de los hombres en trabajos iguales es de entre 15 y $20 \%$ en promedio.

En el contexto latinoamericano son pocas las mujeres que se han posicionado en las esferas más altas de la jerarquía organizacional. De acuerdo con la Organización Internacional del Trabajo ${ }^{3}$ (OIT) (2017), la participación de la mujer en la gestión empresarial y la toma de decisiones podría mejorar el rendimiento financiero. En la misma línea, el Banco Interamericano de Desarrollo (2015) afirma que aquellas empresas que cuentan con participación femenina en posiciones de liderazgo tienen una rentabilidad superior al $44 \%$ en comparación con aquéllas que no cuentan con mujeres. De acuerdo con dicho informe, el porcentaje de mujeres a cargo de una presidencia o dirección oscila entre el $1 \%$ y $3 \%$. La CEPAL manifiesta que la ausencia de mujeres en puestos directivos es un problema que afecta a la economía en general (CEPAL, 2015). En un estudio realizado por dicha organización, en el cual se analizó la situación de las mujeres en 72 grandes compañías en Latinoamérica, a finales de 2014, solo el $4.2 \%$ de éstas contaba con mujeres en el puesto de dirección general o presidente, mientras que la tasa de participación en el equipo directivo fue de un $8 \%$. En la figura 1 se observa que la participación de mujeres tanto en el consejo de administración como en el equipo directivo en Latinoamérica. Se observa que la representación de mujeres mexicanas en el consejo de administración y equipo directivo es de $10.2 \%$ y $6 \%$ respectivamente, por encima de Argentina, Brasil, Chile y Ecuador, mientras que países como Colombia, Perú y Uruguay registran una mayor participación.

\footnotetext{
${ }^{3}$ OIT: Única agencia 'tripartita' de la ONU, la OIT reúne a gobiernos, empleadores y trabajadores de 187 Estados miembros a fin de establecer las normas del trabajo, formular políticas y elaborar programas promoviendo el trabajo decente de todos, mujeres y hombres.
} 
Figura 1. Porcentaje de mujeres en puestos de alta dirección en empresas de América Latina

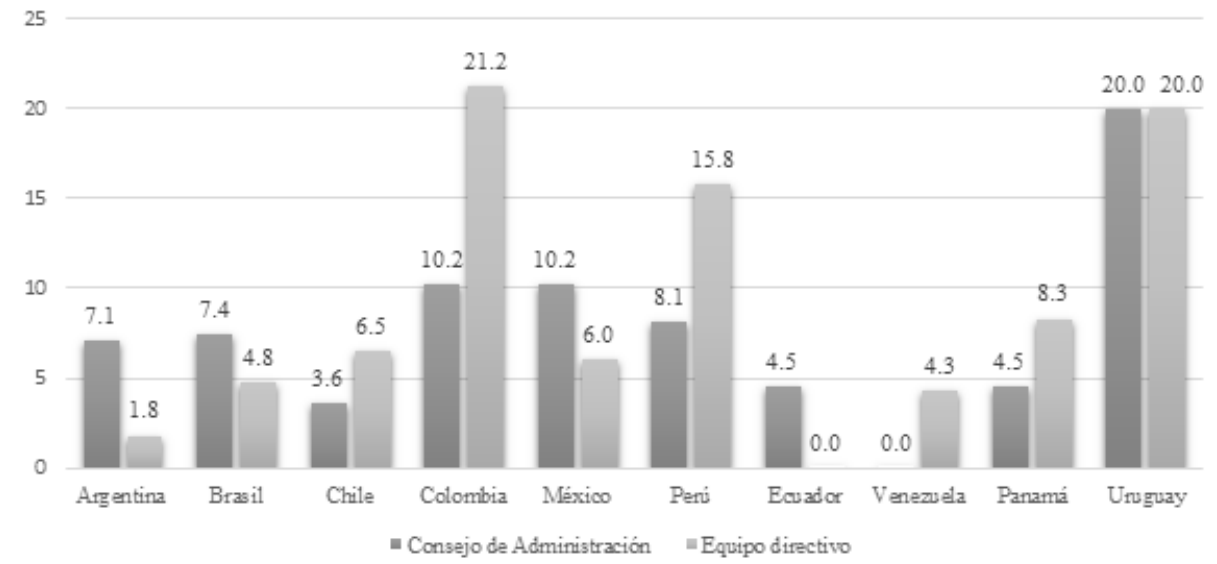

Fuente: CEPAL (2015).

Por su parte, un estudio realizado por el Credit Suisse (2016) sostiene que el porcentaje de participación de mujeres en el consejo de administración en Chile y Brasil es del $8.5 \%$ y $7.1 \%$ respectivamente, mientras que México se encuentra por debajo de éstos porcentajes con un $5.7 \%$. Estas tasas de participación hacen evidente el fenómeno conocido como techo de cristal, el cual es un techo invisible en el que las mujeres encuentran mayores obstáculos para alcanzar la cúspide organizacional (Bruckmüller Branscombe, 2010). Como consecuencia Camarena y Saavedra (2018) encontraron en un análisis de 107 empresas que cotizan en la BMV, que la suma total de los ejecutivos que forman parte del consejo de administración es de 1,426 personas de las cuales solo 65 son mujeres, lo que apenas representa un $4.56 \%$ del total.

\subsection{Hipótesis de Estudio}

\subsubsection{La diversidad de género y el nivel de endeudamiento}

La diversidad de género en el consejo de administración mejora la calidad de los debates entre los consejeros, incrementa la capacidad en la toma de decisiones e incide favorablemente en el desempeño empresarial (Gul, Srinidhi Ng, 2011). En la misma línea, Huse y Solberg (2006), McInerney-Lacombe et al. (2008) y Briano-Turrent (2014) sostienen que la diversidad de género en posiciones estratégicas incrementa la calidad en las deliberaciones del consejo, motiva a una comunicación efectiva e incrementa la transparencia en las prácticas de gobierno corporativo. La teoría de agencia afirma que el cumplimiento de los objetivos corporativos requiere contar con las personas, estructura y recursos adecuados que fundamenten la toma de decisiones y el uso eficiente de los recursos, y la diversidad de género constituye un medio de independencia en el consejo que motiva a mejores resultados empresariales (Ganga-Contreras et al., 2015). El objetivo de la teoría de agencia es describir la relación entre el principal (dueño de la empresa) y el agente, a quien le delega el trabajo y objetivos de la empresa (Zapata-Rotundo Hernández-Arias, 2010). Generalmente existe un conflicto de intereses entre los gerentes, accionistas y tenedores de bonos, los cuales eligen la propiedad de sus acciones de la organización, la mezcla de la deuda externa, el financiamiento del capital y los dividendos que ayuden a disminuir los costos de dichos conflictos. Es aquí en donde el nivel de endeudamiento toma relevancia para la teoría de la agencia, ya que mediante el empleo de la deuda se reduce el conflicto 
636 REMEF (The Mexican Journal of Economics and Finance)

Diversidad de género en posiciones estratégicas y el nivel de endeudamiento: Evidencia en empresas cotizadas mexicanas

de intereses y la dependencia de capital externo para la empresa (Crutchley Hansen, 1989).

Otra teoría que soporta la diversidad de género en el consejo, es la teoría de la masa crítica, que hace referencia a la representación sustantiva de la mujer en órganos de decisión. Kanter (1977) afirma que las dinámicas y procesos internos del grupo dependen del porcentaje de representación del grupo minoritario, y define cuatro tipos de proporciones: a) grupo uniforme (100\%-0\%); b) grupo sesgado (token), (85\%-15\%); c) grupo inclinado (65\%-35\%), y d) grupo balancedo (50\%-50\%). De acuerdo a la citada autora, las mujeres que pertenecen a un grupo sesgado se enfrentan a presiones de desempeño lo que podría limitar su visibilidad para pasar desapercibidas y mezclarse con el grupo dominante. En cambio, un equipo balanceado entre hombres y mujeres incide en la cultura del grupo y se obtienen mejores resultados financieros (González, 2016). Por ejemplo los consejos de administración con una participación de mujeres de entre un 20\%-40\% alcanzan discusiones productivas y afectan positivamente el desempeño financiero (Joecks, Pull Vetter, 2013; Konrad et al., 2008; Torchia et al., 2011).

Si bien diversos estudios sostienen que las mujeres son más aversas al riesgo, que tienen una preferencia hacia estrategias menos agrasivas e invierten de forma más sustentable (Croson Gneezy, 2009; Apesteguia et al. 2012 y Charness Gneezy 2012), también podrían agregar más valor al consejo proveyendo de nuevas perspectivas y discusiones productivas, lo que a su vez favorece el desempeño empresarial (Konrad Kramer, 2006). Por ejemplo, el Credit Suisse (2012) evidencia que aquellas compañías que cuentan con mujeres en su equipo directivo, tienen una mayor rentabilidad financiera, más valor en sus acciones y mayor crecimiento a largo plazo. Gordini y Racanti (2017) sugieren que la diversidad de género motiva a mayores ganancias, por lo que las empresas deben enfocarse en buscar un equilibrio entre hombres y mujeres. Terjesen et al. (2015) analizan de manera empírica si la diversidad de género mejora la independencia y la eficiencia del consejo, y sostienen que aquellas empresas que cuentan con más mujeres en posiciones directivas, tienen un mejor desempeño de mercado y contable.

Con respecto a la influencia de las mujeres en las decisiones de financiamiento, Jeong y Harrison (2017) sostienen que a pesar de que la aversión a la toma de riesgos es mayor en las mujeres, cuando la posición de CEO está a cargo de una mujer podría favorecer el desempeño financiero a largo plazo. En la misma línea, McGuinness, Vieito y Wang (2017) evidencian un mejor desempeño en materia de responsabilidiad social corporativa en aquellas empresas que cuentan con mujeres en posiciones de CEO, además de una relación positiva con el endeudamiento y el retorno del capital en empresas cotizadas. Por su parte, Francis, Hasan y Wu (2013) asocian la presencia mujeres en posiciones estratégicas con una mayor probabilidad de incrementar las ganancias y tienden a reducir el nivel de endeudamiento en comparación con las posiciones ocupadas por hombres, debido a que las mujeres son menos propensas a manipular las ganacias de la empresa y a tomar menos riesgos. Por su parte, Coleman y Robb (2009) sugieren que las mujeres tienen una preferencia por las fuentes de deuda y capital, tanto para el capital inicial como para las inversiones. Con base en lo anterior se establecen las siguientes hipótesis:

$H_{1 A}$ : La diversidad de género en el consejo y equipo directivo incide positivamente en el nivel de endeudamiento en empresas cotizadas mexicanas.

$H_{2 A}$ : La diversidad de género en el consejo y equipo directivo incide positivamente en la rentabilidad de empresas cotizadas mexicanas. 
Las diferencias de género en actitudes hacia el riesgo han sido ampliamente estudiadas y se afirma que las mujeres generalmente son más aversas al riesgo en comparación con los hombres.. Por ejemplo, Huang y Kisgen (2013) evidencian que cuando las mujeres forman parte del equipo directivo, hay menos probabilidad de realizar adquisiciones significativas o emitir deuda a largo plazo, aunque obtienen una mayor rentabilidad en comparación con el género masculino. Del mismo modo, Francis et al. (2014) afirman que las mujeres que ocupan la dirección de finanzas tienen una preferencia por decisiones de inversión y financieras menos riesgosas, mientras que Liu, Wei y Xie (2016) sostienen que las mujeres son menos propensas a tomar riesgos y a manipular los ingresos y resultados financieros en comparación con los hombres. Otros estudios ponen de manifiesto una relación negativa entre la participación de mujeres en posiciones directivas y el desempeño financiero de la empresa (Vithessonthi Tongurai, 2015). Por ejemplo, Mínguez-Vera y Martin (2011) sostienen que la participación de la mujer en el consejo genera un impacto negativo en el desempeño empresarial, debido a la implementación de estrategias menos riesgosas y una actitud conservadora en las decisiones de inversión. De acuerdo con Adams y Ferreira (2009), la presencia femenina tiene un impacto significativo en las decisiones del consejo y afirman que su influencia en el desempeño empresarial es negativa, y que el mandato basado en una cuota de género en posiciones directivas puede generar una disminución en el valor de la empresa. Pfeifer y Wagner (2014), por su parte, revelan menores niveles de productividad y rentabilidad en empresas con una mayor cantidad de mujeres. Jeong y Harrison (2017) sostienen que la representación femenina en puestos directivos inciden positivamente con el desempeño financiero a largo plazo de la empresa, más sin embargo, observan una relación negativa con el rendimiento del mercado accionario a corto plazo. Bajo estas circunstancias, existen estudios que basados en las diferencias de género y la tolerancia al riesgo, sugieren que las mujeres que se desempeñan en posiciones de CEO, evalúan de forma más conservadora los riesgos y por ende, mantienen mayores niveles de capital social y minimizan las probabilidades de quiebra (Palvia, Vähämaa Vähämaa 2015).

En la misma línea, Pucheta y Sánchez (2013) evidencian que las mujeres inciden negativamente en el nivel de endeudamiento y en la rentabilidad, mientras que Loukil y Yousfi (2015) afirman que no existe una relación significativa entre la presencia de mujeres directivas y la toma de riesgos estratégicos y financieros, además de que los inversionistas tienden a no invertir en empresas con mayor diversidad de género. Saeed y Sameer (2017) sostienen que tanto el coeficiente de riesgo como el de apalancamiento se ven afectados de manera negativa y significativa con la participación de las mujeres como directoras, lo que implica que la volatilidad de los ingresos y el financiamiento de la deuda representen una limitante para la organización. Con base en las premisas anteriores, se establecen la siguientes hipotesis:

$H_{2 A}$ : La diversidad de género en el consejo de administración y equipo directivo afecta negativamente el nivel de endeudamiento en empresas cotizadas mexicanas.

$H_{2 B}$ : La diversidad de género en el consejo y equipo directivo afecta negativamente la rentabilidad de empresas cotizadas mexicanas.

\section{Metodología de Investigación}

El trabajo utiliza un panel de datos no balanceado controlando los efectos fijos por sector industrial y año de estudio con el objetivo de analizar la relación entre la diversidad de género y el nivel de endeudamiento de las empresas cotizadas en México. Se realiza además una regresión Logit para confirmar los resultados obtenidos en el análisis regresión múlti- 
638 REMEF (The Mexican Journal of Economics and Finance)

Diversidad de género en posiciones estratégicas y el nivel de endeudamiento: Evidencia en empresas cotizadas mexicanas

ple. Para ello se clasificó a las empresas más endeudadas vs menos endeudadas, categorizando a la variable dependiente de endeudamiento como una variable dicotómica, en donde 1 es para aquellas empresas con un ratio de endeudamiento mayor a la mediana por año de estudio y de 0 en caso de que la empresa se encuentre por debajo de ese valor. Se construyó una base de datos que integra variables de género, composición del consejo y variables financieras, las cuales fueron extraídas de forma manual de los informes anuales de las empresas que cotizan en la Bolsa Mexicana de Valores. A través del análisis de contenido, se realizó la búsqueda de palabras clave tres veces para localizar y garantizar la calidad de la información. También se realizó un estudio de correlación para asegurar la ausencia de correlación entre las variables y se trataron los valores extremos de las variables financieras truncando su valor al percentil 2. Además, se calcularon los Factores de Inflación de la Varianza (FIV) en el análisis de regresión múltiple para identificar posibles problemas de colinealidad entre las variables de estudio.

\subsection{Muestra de estudio}

La tabla 1 presenta la distribución de la muestra de estudio por sector industrial, la cual se integra por un total de 874 observaciones-año, de un total de 98 empresas de los sectores de alimentos, bienes y equipo, construcción, energía, materiales, salud, telecomunicaciones, servicios y bienes de consumo, suministros, tecnologías de la información y transportes. El $22 \%$ de las observaciones se ubica en el sector de materiales, el $17.3 \%$ en el sector de construcción y el $13.8 \%$ pertenece al sector de alimentos. De la población total se han excluido las empresas que pertenecen al sector financiero dado que su regulación difiere del resto de empresas, así como aquellas empresas que no publican sus informes anuales o cuya información no está disponible durante el periodo de análisis 2004-2016.

Tabla 1. Medición de errores en la fase A del pronóstico del IPC.

\begin{tabular}{lcccc}
\hline \multicolumn{1}{c}{ Sector industrial } & $\begin{array}{c}\text { Porcentaje } \\
\text { empresas }\end{array}$ & N empresas & $\begin{array}{c}\text { Porcentaje } \\
\text { observaciones }\end{array}$ & N observaciones \\
\hline Alimentos, & $11.20 \%$ & 11 & $13.80 \%$ & 121 \\
bebidas y tabaco & $7.10 \%$ & 7 & $8.50 \%$ & 74 \\
Bienes de equipo & $17.30 \%$ & 17 & $17.30 \%$ & 151 \\
Construcción & $2.00 \%$ & 2 & $2.20 \%$ & 19 \\
Suministros y servicios comerciales & $6.10 \%$ & 6 & $6.20 \%$ & 54 \\
Transportes & $22.50 \%$ & 22 & $22.00 \%$ & 192 \\
Materiales & $18.50 \%$ & 18 & $14.50 \%$ & 127 \\
Servicios y bienes de consumo no básico & $8.20 \%$ & 8 & $10.90 \%$ & 95 \\
Telecomunicaciones & $5.10 \%$ & 5 & $3.90 \%$ & 34 \\
Salud & $1.00 \%$ & 1 & $0.60 \%$ & 5 \\
Energía & $1.00 \%$ & 1 & $0.20 \%$ & 2 \\
Tecnología de & & & $\mathbf{1 0 0 \%}$ & $\mathbf{8 7 4}$ \\
la información & $\mathbf{1 0 0 \%}$ & $\mathbf{9 8}$ & \\
\hline
\end{tabular}

Fuente: Elaboración propia con datos de extraídos de la Bolsa Mexicana de Valores (2017).

\subsection{Modelos Econométricos}

Para analizar la influencia que tiene la participación femenina en posiciones directivas sobre el nivel de endeudamiento en empresas cotizadas mexicanas, se establecen tres modelos econométricos utilizando un panel de datos no balanceado con efectos fijos que analizan la relación entre la diversidad de género en posiciones estratégicas y el nivel desempeño financiero (nivel de endeudamiento y rentabilidad). La ecuación 1 hace referencia a la diversidad de género en el consejo y su impacto en las decisiones de endeudamiento. Particularmente, la variable dependiente de endeudamiento se mide a través de dos ratios: 
el endeudamiento a largo plazo y el endeudamiento total, las variables independientes se centran en si una mujer ocupa la posición de COB (variable dicotómica) y el porcentaje de mujeres en el consejo de administración. Las variables de control se centran en el tamaño del consejo, la independencia del consejo, ROA, tamaño, edad de la empresa, sector industrial y año de estudio. La ecuación 2 analiza la relación entre la participación femenina en la posición de director general (CEO) o el porcentaje de mujeres en el equipo directivo y el nivel de endeudamiento. La variable dependiente de endeudamiento se mide a través de dos ratios: el endeudamiento a largo plazo y el endeudamiento total, las variables independientes se miden a través de dos variables de género: si una mujer ocupa la posición de CEO (variable dicotómica) y el porcentaje de mujeres en el equipo directivo. Las variables de control se refieren al tamaño del consejo, la independencia del consejo, ROA, tamaño, edad de la empresa, sector industrial y año de estudio. La ecuación 3 analiza la relación entre la participación femenina en el consejo de administración y la rentabilidad, mientras que la ecuación 4 asocia la variable género en el equipo directivo y el ROA. La variable dependiente se refiere al ratio de rendimiento de la inversión, las variables independientes se refieren al género del COB, CEO y porcentaje de mujeres en el consejo y equipo directivo. Las variables de control se refieren al tamaño del consejo, la independencia del consejo, endeudamiento, tamaño, edad de la empresa, sector industrial y año de estudio. La información relacionada a cada una de las variables de estudio han sido obtenidas de forma manual de cada uno de los informes anuales de las empresas analizadas. La tabla 2 describe la medición de cada una de las variables de estudio.

$$
\begin{aligned}
& \text { Leverage(largoplazoytotal })_{i, t}=\alpha_{0}+\alpha_{1} \text { GenderBoard }_{i, t}+\alpha_{2} \text { Control }_{i, t}+\mu_{i, t} \\
& \text { Leverage(largoplazoytotal })_{i, t}=\alpha_{0}+\alpha_{1} \text { GenderExTeam }_{i, t}+\alpha_{2} \text { Control }_{i, t}+\mu_{i, t} \\
& R O A_{i, t}=\alpha_{0}+\alpha_{1} \text { GenderBoard }_{i, t}+\alpha_{2} \text { Control }_{i, t}+\mu_{i, t} \\
& \text { ROA }_{i, t}=\alpha_{0}+\alpha_{1} \text { GenderExTeam }_{i, t}+\alpha_{2} \text { Control }_{i, t}+\mu_{i, t}
\end{aligned}
$$

En la sección de análisis empíricos adicionales se adopta el modelo de regresión Logit para confirmar los resultados referidos al nivel de endeudamiento, calculando una variable dicotómica que toma el valor de 1 cuando la empresa obtiene un ratio de endeudamiento mayor al valor de la mediana y de 0 en caso contrario en cada uno de los años de estudio. La variable dependiente toma el valor de 1 si la variable de endeudamiento a largo plazo o total está por encima de la mediana y de 0 en caso contrario. Las variables independientes se refieren a la dimensión de género descritas en el párrafo anterior, mientras que las variables de control que se integran al modelo son el tamaño del consejo, la independencia del consejo, ROA, tamaño, edad de la empresa, sector industrial y año de estudio. Las ecuaciones 5 y 6 que analizan esta relación a través de un modelo Logit son:

$$
\begin{aligned}
& \text { Leverage }\left(\text { msendeudadas }_{i, t}=\alpha_{0}+\alpha_{1} \text { GenderBoard }_{i, t}+\alpha_{2} \text { Control }_{i, t}+\mu_{i, t}\right. \\
& \text { Leverage }\left(\text { msendeudadas }_{i, t}=\alpha_{0}+\alpha_{1} \text { GenderExTeam }_{i, t}+\alpha_{2} \text { Control }_{i, t}+\mu_{i, t}\right.
\end{aligned}
$$

Donde:

$\alpha_{2}\left(\right.$ Controls $\left._{i t}\right)=$ Conjunto de variables de control: $\mathrm{t}$ el tamaño del consejo, la independencia del consejo, ROA, tamaño, edad de la empresa, sector industrial y año de estudio. $\mu_{i t}=$ Término de error. 
640 REMEF (The Mexican Journal of Economics and Finance)

Diversidad de género en posiciones estratégicas y el nivel de endeudamiento: Evidencia en empresas cotizadas mexicanas

\subsection{Variables de estudio}

La tabla 2 describe las variables de estudio, las cuales se han dividido en cuatro grupos: 1) variables dependientes (endeudamiento total y endeudamiento a largo plazo), 2) variables independientes (diversidad de género en el consejo), 3) variables de control referidas a la composición de consejo, y 4) variables de control relacionadas con las características de las empresas analizadas.

Tabla 2. Definición y medición de las variables de estudio.

\begin{tabular}{|c|c|c|}
\hline Variables & Medición & Fuente \\
\hline \multicolumn{3}{|c|}{ Variables dependientes } \\
\hline Endeudamiento total & Pasivos Totales/ Activos Totales & Jeong y Harrison (2017). \\
\hline Endeudamiento a largo plazo & Pasivo a largo plazo/ Capital Contable & $\begin{array}{l}\text { Francis, Hasan y Wu (2013), } \\
\text { Miller, Le-Bretton-Miller y Lester } \\
\text { (2013), Pucheta y Sánchez (2013). }\end{array}$ \\
\hline$R O A$ & $\begin{array}{l}\text { Rendimiento de la inversión: Ingresos } \\
\text { operativos/Valor en libros de los activos. }\end{array}$ & Pucheta y Sánchez (2013). \\
\hline \multicolumn{3}{|c|}{ Variables independientes } \\
\hline COB Mujer & $\begin{array}{l}\text { Variable dicotómica que toma el valor de } \\
1 \text { si una mujer ocupa la posición de COB } \\
\text { y } 0 \text { en caso contrario. }\end{array}$ & \\
\hline$\%$ mujeres consejo & $\begin{array}{l}\text { \% de mujeres en el consejo = Número de } \\
\text { mujeres que forman parte del } \\
\text { consejo/Total de miembros del consejo. }\end{array}$ & \\
\hline CEO Mujer & $\begin{array}{l}\text { Variable dicotómica que toma el valor de } \\
1 \text { si una mujer ocupa la posición de CEO } \\
\text { y } 0 \text { en caso contrario. }\end{array}$ & $\begin{array}{l}\text { Mateos de Cabo, Gimeno y Escot } \\
(2010) \text {. }\end{array}$ \\
\hline$\%$ mujeres equipo directivo & $\begin{array}{l}\text { \% de mujeres en el equipo directivo = } \\
\text { Número de mujeres que participan en el } \\
\text { equipo directivo/ Total de miembros del } \\
\text { equipo directivo. }\end{array}$ & $\begin{array}{l}\text { Mateos de Cabo, Gimeno y Escot } \\
(2010) \text {. }\end{array}$ \\
\hline \multicolumn{3}{|c|}{ Variables de control (Consejo de administración) } \\
\hline$B S$ & $\begin{array}{l}\text { Tamaño del consejo: número de cargos } \\
\text { ocupados por miembros propietarios } \\
\text { (excluye suplentes). }\end{array}$ & $\begin{array}{l}\text { Mateos de Cabo, Gimeno y Escot } \\
\text { (2010); Pucheta y Sánchez (2013). }\end{array}$ \\
\hline$B I$ & $\begin{array}{l}\text { Independencia del Consejo: Número de } \\
\text { consejeros independientes/consejeros } \\
\text { totales. }\end{array}$ & $\begin{array}{l}\text { Mateos de Cabo, Gimeno y Escot } \\
\text { (2010), Pucheta y Sánchez (2013). }\end{array}$ \\
\hline Duality & $\begin{array}{l}\text { Variable dicotómica que toma el valor de } \\
1 \text { si el presidente del Consejo y Director } \\
\text { General son la misma persona y de } 0 \text { en } \\
\text { caso contrario. }\end{array}$ & Pucheta y Sánchez (2013). \\
\hline \multicolumn{3}{|c|}{ Variables de control (características de la empresa) } \\
\hline Tam_empresa & $\begin{array}{l}\text { Tamaño de la empresa: Logaritmo natural } \\
\text { de los activos totales. }\end{array}$ & $\begin{array}{l}\text { Ali, Ng y Kulik (2014), Mateos de } \\
\text { Cabo, Gimeno y Escot (2010). }\end{array}$ \\
\hline Age & $\begin{array}{l}\text { Edad de la empresa: Años desde la } \\
\text { fundación de la empresa. }\end{array}$ & Ali, Ng y Kulik (2014). \\
\hline Listed years & $\begin{array}{l}\text { Número de años que la empresa } \\
\text { tiene cotizando en la BMV. }\end{array}$ & $\begin{array}{l}\text { Ali, Ng y Kulik (2014), Pucheta y } \\
\text { Sánchez (2013). }\end{array}$ \\
\hline Sector & $\begin{array}{l}\text { Sector industrial al que pertenece la } \\
\text { empresa. }\end{array}$ & Bolsa Mexicana de Valores (2017). \\
\hline Year & $\begin{array}{l}\text { Año de estudio: Variable dicotómica por } \\
\text { cada año de estudio. }\end{array}$ & \\
\hline
\end{tabular}

Fuente: Elaboración propia basada en la literatura previa. 


\section{Resultados}

\subsection{Análisis Descriptivo}

La tabla 3, muestra los estadísticos descriptivos de las variables de estudio. Las variables dependientes muestran que las empresas bajo estudio registran un promedio de endeudamiento total de $0.53 \mathrm{y}$ de endeudamiento a largo plazo de 0.28 lo que nos sugiere que las empresas cotizadas en México no adoptan estrategias agresivas de apalancamiento. El $51.49 \%$ de las empresas se encuentran endeudadas a largo plazo por encima de la mediana, mientras que el $50.23 \%$ del total de empresas tienen ratios de endeudamiento total por encima de la media. En relación a las variables de género en posiciones estratégicas, se observa que solamente 3 mujeres ocupan la posición de presidente del consejo (COB) y 3 mujeres como CEOs, lo que representa un escaso $0.34 \%$. Por su parte, el porcentaje de mujeres en el consejo de administración representa el $3.74 \%$, mientras que el porcentaje de mujeres en el equipo directivo alcanza apenas en promedio un $4 \%$. Estos datos demuestran la escasa participación de mujeres en cargos de alta responsabilidad en las empresas de mayor tamaño en México, lo cual inhibe la injerencia de éstas en la toma de decisiones financieras en la empresa. También es importante describir otras características del consejo, como es su tamaño, el cual obtiene un promedio de 12 miembros, mientras que las empresas han impulsado su independencia con un $47 \%$ de consejeros independientes. La práctica de dualidad COB-CEO es adoptada por 485 empresas, es decir, en el $55.5 \%$ de las observaciones, el presidente del consejo de administración (COB) ocupa también la posición de director general (CEO). Finalmente, se observa que las empresas obtienen un tamaño promedio de 17.13, una edad promedio de 30 años, una antigüedad de 18 años cotizando en bolsa y una rentabilidad de 0.04 .

Tabla 3. Estadísticos descriptivos de las variables de estudio.

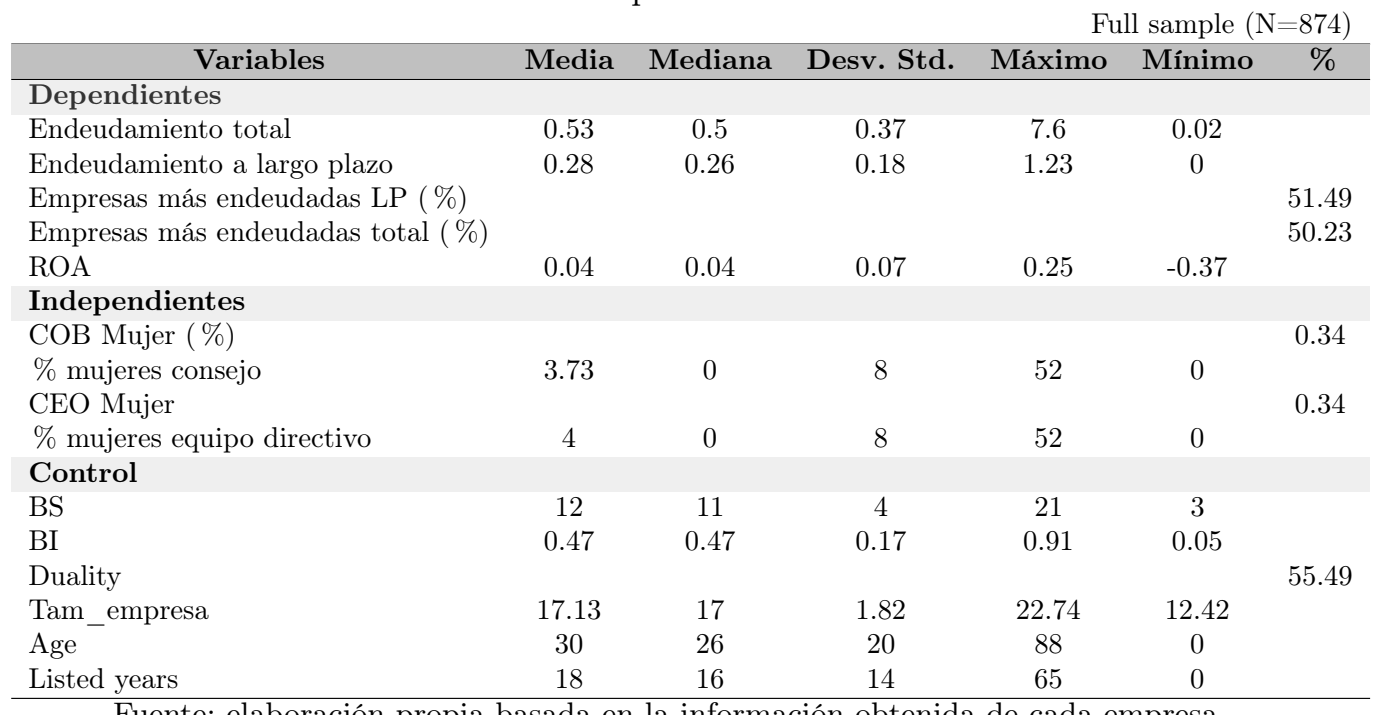

Fuente: elaboración propia basada en la información obtenida de cada empresa.

La tabla 4, describe por año de estudio la evolución de la participación femenina en el consejo de administración y equipo directivo. Como se puede observar solo se tiene evidencia de participación femenina en la posición de COB de una mujer en los años 2008, 2015 y 2016, mientras que en la posición de CEO solo se registran 3 casos en los años 2005, 2006 y 2016. En comparación con el 2004, la participación femenina paso del 
642 REMEF (The Mexican Journal of Economics and Finance)

Diversidad de género en posiciones estratégicas y el nivel de endeudamiento: Evidencia en empresas cotizadas mexicanas

$2 \%$ al $4 \%$. El porcentaje máximo de participación femenina como miembros del consejo es de $5.29 \%$ en 2004 y un promedio de $3.85 \%$ en el periodo 2004-2016. Finalmente, la presencia de mujeres en el equipo directivo es de un promedio de $3.16 \%$ durante el periodo de análisis y de $4.54 \%$ en 2014. Los resultados anteriores nos sugieren una escasa representación de las mujeres en la cúspide de las empresas cotizadas mexicanas, aunque se ha registrado un ligero incremento en los últimos cinco años.

Tabla 4. Participación femenina en posiciones estratégicas

\begin{tabular}{|c|c|c|c|c|}
\hline \multirow{2}{*}{ Año } & \multicolumn{4}{|c|}{ Variables de género } \\
\cline { 2 - 5 } & COB mujer & CEO mujer & $\begin{array}{c}\text { \% mujeres } \\
\text { consejo }\end{array}$ & $\begin{array}{c}\text { \% mujeres } \\
\text { equipo } \\
\text { directivo }\end{array}$ \\
\hline 2004 & 0 & 0 & 5.29 & 2 \\
2005 & 0 & 1 & 3,63 & 2.59 \\
2006 & 0 & 1 & 4.56 & 2.62 \\
2007 & 0 & 0 & 3.9 & 2.1 \\
2008 & 1 & 0 & 3.78 & 1.92 \\
2009 & 0 & 0 & 3.33 & 2 \\
2010 & 0 & 0 & 2.94 & 2.27 \\
2011 & 0 & 0 & 3.4 & 3.85 \\
2012 & 0 & 0 & 3.47 & 4.36 \\
2013 & 0 & 0 & 3.52 & 4.43 \\
2014 & 0 & 0 & 3.56 & 4.54 \\
2015 & 1 & 0 & 3.98 & 4.33 \\
2016 & 1 & 1 & 4.46 & 4.01 \\
\hline Total & $\mathbf{3}$ & $\mathbf{3}$ & $\mathbf{3 . 8 5}$ & $\mathbf{3 . 1 6}$ \\
\hline
\end{tabular}

Fuente: elaboración propia basada en la información obtenida de cada empresa.

\subsection{Análisis de Correlación}

La tabla 5 muestra el análisis de correlación entre las variables de estudio. Se observa que las correlaciones significativas y positivas se encuentran entre las siguientes variables: endeudamiento total con endeudamiento a largo plazo $(\mathrm{p}=0.01)$, dualidad COB y endeudamiento total $(\mathrm{p}=0.01)$, tamaño del consejo, dualidad COB-CEO y tamaño de la empresa con endeudamiento a largo plazo $(\mathrm{p}=0.01), \%$ de mujeres en el equipo directivo, dualidad COB-CEO, edad de la empresa y años cotizados en bolsa con el porcentaje de mujeres en el consejo $(\mathrm{p}=0.01)$, porcentaje de mujeres en el equipo directivo y tamaño de la empresa con CEO mujer, dualidad COB-CEO con el porcentaje de mujeres en equipo directivo, tamaño, edad y años cotizadas en bolsa de la empresa con tamaño del consejo $(\mathrm{p}=0.01)$, edad de la empresa y ROA con el tamaño de la empresa $(\mathrm{p}=0.01)$, y años cotizados con la edad de la empresa $(\mathrm{p}=0.01)$. Por su parte las correlaciones negativas y significativas son: la rentabilidad con el endeudamiento total $(\mathrm{p}=0.01)$, la independencia del consejo con el porcentaje de mujeres en el consejo $(\mathrm{p}=0.01)$, el tamaño del consejo, tamaño de la empresa, la edad de la empresa y los años cotizando con el porcentaje de mujeres el equipo directivo $(\mathrm{p}=0.01)$, la independencia del consejo y la dualidad COBCEO con el tamaño del consejo $(\mathrm{p}=0.01)$ y el ROA con la dualidad COB-CEO $(\mathrm{p}=0.01)$. Los resultados anteriores no muestran una correlación significativa entre las variables de diversidad de género y las decisiones de endeudamiento en empresas cotizadas mexicanas, lo cual podría ser explicado por la escasa presencia de mujeres en estas posiciones. No se evidencian coeficientes altos de correlación entre las variables de estudio. 


\begin{tabular}{|c|c|c|c|c|c|c|c|c|c|c|c|c|}
\hline Variable & End. Total & End. LP & Tabla & Análisis & $\begin{array}{c}\text { de corre } \\
\text { CEO mujer }\end{array}$ & lación bi $_{\% \text { muj EqDiir }}$ & $\underset{\text { BS }}{\text { varia }}$ & da. & Duality & Tam_emp & Age & Listed years \\
\hline End. LP & $0.28^{* *}$ & & & & & & & & & & & \\
\hline COB Muj & -0.05 & -0.04 & & & & & & & & & & \\
\hline$\%$ mujeres consejo & -0.02 & 0.01 & 0.05 & & & & & & & & & \\
\hline CEO Muj & 0 & -0.05 & 0 & $0.07^{*}$ & & & & & & & & \\
\hline \% Muj EqDi & 0.05 & -0.06 & -0.03 & $0.18^{* *}$ & $0.14^{* *}$ & & & & & & & \\
\hline BS & -0.04 & $0.14^{* *}$ & -0.01 & $0.07^{*}$ & 0.02 & $-0.18^{* *}$ & & & & & & \\
\hline $\mathrm{BI}$ & -0.03 & $-0.15 * *$ & 0.04 & $-0.18^{* *}$ & 0.03 & 0.02 & $-0.27^{* *}$ & & & & & \\
\hline Duality & $0.14^{* *}$ & $0.17^{* *}$ & -0.07 & $0.10^{* *}$ & -0.07 & $0.12^{* *}$ & $-0.10^{* *}$ & 0.05 & & & & \\
\hline Tam_emp & -0.03 & $0.12^{* *}$ & 0.01 & -0.05 & $0.10^{* *}$ & $-0.10^{* *}$ & $0.30^{* *}$ & -0.01 & -0.01 & & & \\
\hline Age ${ }^{-}$ & -0.01 & $-0.12^{* *}$ & -0.04 & $0.16^{* *}$ & 0.03 & $-0.06^{* *}$ & $0.10^{* *}$ & 0.05 & 0.06 & $0.12^{* *}$ & & \\
\hline Listed years & -0.01 & -0.06 & -0.05 & $0.12^{* *}$ & -0.01 & $-0.06^{* *}$ & $0.18^{* *}$ & 0.06 & 0.05 & -0.04 & $0.78^{* *}$ & \\
\hline $\mathrm{ROA}$ & $-0.41^{* *}$ & $-0.18^{* *}$ & 0.03 & 0.01 & 0.01 & $-0.11^{*}$ & $-0.14^{* *}$ & -0.03 & $-0.09 * *$ & $0.17^{* *}$ & 0.01 & 0.01 \\
\hline
\end{tabular}

Fuente: elaboración propia basada en la información obtenida de cada empresa.

Nota: Las correlaciones son significativas a los niveles de $0.05^{*}$ y $0.01^{* *}$. Las definiciones y medición de las variables de estudio se pueden consultar en la tabla 2.

\subsection{Análisis de Regresión Múltiple}

\subsubsection{Diversidad de género y nivel de endeudamiento}

En esta sección se describen los resultados obtenidos al analizar la relación entre la diversidad de género y el nivel de endeudamiento. Se inicia el análisis con un modelo de regresión utilizando el método de mínimos cuadrados ordinarios para probar las hipótesis que establece que la participación de mujeres reduce el nivel de endeudamiento total y a largo plazo. Se controlan los efectos fijos de sector industrial y año de estudio con variables dicotómicas. En los modelos 1 y 2 se incluyen como variables explicativas la diversidad de género en el consejo y equipo directivo, mientras que en los modelos 3 y 4 se agregan las variables de composición del consejo como son su tamaño, independencia y dualidad COB-CEO. El modelo 1 evidencia que cuando la posición de COB es ocupada por una mujer incide negativamente en el nivel de endeudamiento a largo plazo $(\mathrm{p}=0.01)$, mientras que el porcentaje de mujeres en el consejo lo incrementa $(\mathrm{p}=0.05)$. Empresas de mayor tamaño motivan a un mayor endeudamiento a largo plazo, mientras que los sectores de construcción, transportes y servicios de telecomunicaciones obtienen los mayores ratios de endeudamiento a largo plazo.

El modelo 2 pone de manifiesto que las mujeres CEOs reducen el nivel de endeudamiento a largo plazo ( $\mathrm{p}=0.01)$, mientras que el tamaño de la empresa lo aumenta. El modelo 3, confirma que las mujeres CEOs disminuyen el nivel de endeudamiento, mientras que las mujeres que forman parte del consejo lo incrementan. El tamaño del consejo y la dualidad COB-CEO aumentan el endeudamiento a largo plazo mientras que la independencia del consejo lo disminuye. El modelo 4 demuestra que las mujeres CEOs y que forman parte del equipo directivo no tienen influencia significativa en el nivel de endeudamiento. Por su parte, el tamaño del consejo y la dualidad COB-CEO aumentan el endeudamiento a largo plazo, mientras que la independencia del consejo lo disminuye. Los resultados nos sugieren que a pesar de la escasa participación femenina en posiciones estratégicas, su influencia es significativa cuando éstas forman parte del consejo de administración.

Tabla 6. Regresión diversidad de género y nivel de endeudamiento a largo plazo

Esta tabla presenta el análisis de regresión múltiple utilizando el método de Huber-White con errores estándar corregidos. La variable dependiente se refiere al Endeudamiento a Largo Plazo (LLEV) en el año t. Las variables independientes se refieren a la diversidad de género en el Consejo de Administración (modelos 1 y 3 ) y la presencia de mujeres en el equipo directivo (modelos 2 y 4). Las variables de control incluidas en el modelo son el ROA, Tamaño de la Empresa, Edad de la Empresa, Tamaño del Consejo, Independencia del Consejo y Dualidad COB-CEO. Además, se incluyeron variables dummies para controlar por sector industrial y año de estudio. 
644 REMEF (The Mexican Journal of Economics and Finance)

Diversidad de género en posiciones estratégicas y el nivel de endeudamiento: Evidencia en empresas cotizadas mexicanas

\begin{tabular}{|c|c|c|c|c|}
\hline & $(1)$ & $(2)$ & $(3)$ & $(4)$ \\
\hline Variables & LLEV & LLEV & LLEV & LLEV \\
\hline Mujer COB & $\begin{array}{c}-0.192^{* * *} \\
(-2.59)\end{array}$ & & $\begin{array}{c}-0.149^{* *} \\
(-2.21)\end{array}$ & \\
\hline$\%$ mujeres consejo & $\begin{array}{c}0.121^{* *} \\
-2\end{array}$ & & $\begin{array}{c}0.107^{*} \\
-1.69\end{array}$ & \\
\hline Mujer CEO & & $\begin{array}{c}-0.080 * * * \\
(-2.39)\end{array}$ & & $\begin{array}{c}-0.05 \\
(-0.84)\end{array}$ \\
\hline$\%$ de mujeres equipo directivo & & $\begin{array}{l}0.022 \\
-0.26\end{array}$ & & $\begin{array}{c}0.05 \\
-0.46\end{array}$ \\
\hline $\mathrm{ROA}$ & $\begin{array}{l}-0.001 \\
(-1.04)\end{array}$ & $\begin{array}{l}-0.001 \\
(-1.07)\end{array}$ & $\begin{array}{l}-0.001 \\
(-1.13)\end{array}$ & $\begin{array}{l}-0.001 \\
(-1.15)\end{array}$ \\
\hline Tamaño de la empresa & $\begin{array}{c}0.013^{* * *} \\
-4.13\end{array}$ & $\begin{array}{c}0.014^{* * *} \\
-4.58\end{array}$ & $\begin{array}{c}0.009 * * * \\
-2.56\end{array}$ & $\begin{array}{c}0.009 * * * \\
-2.67\end{array}$ \\
\hline Edad de la empresa & $\begin{array}{c}0 \\
(-1.19)\end{array}$ & $\begin{array}{c}0 \\
(-0.95)\end{array}$ & $\begin{array}{l}-0.001 \\
(-1.60)\end{array}$ & $\begin{array}{c}0 \\
(-1.28)\end{array}$ \\
\hline Tamaño del consejo & & & $\begin{array}{c}0.008^{* * *} \\
-4.79\end{array}$ & $\begin{array}{c}0.008^{* * *} \\
-4.83\end{array}$ \\
\hline Independencia del consejo & & & $\begin{array}{c}-0.088^{* *} \\
(-2.24)\end{array}$ & $\begin{array}{c}-0.096^{* * *} \\
(-2.43)\end{array}$ \\
\hline Dualidad COB-CEO & & & $\begin{array}{c}0.084^{* * *} \\
-6.96\end{array}$ & $\begin{array}{c}0.084^{* * *} \\
-6.89\end{array}$ \\
\hline Sector industrial & Sí & Sí & Sí & Sí \\
\hline Año de estudio & No & No & No & No \\
\hline Constante & $\begin{array}{c}0.024 \\
-0.4\end{array}$ & $\begin{array}{l}0.008 \\
-0.14\end{array}$ & $\begin{array}{l}-0.027 \\
(-0.43)\end{array}$ & $\begin{array}{l}-0.024 \\
(-0.39)\end{array}$ \\
\hline$R^{2}$ ajustado & 0.16 & 0.158 & 0.22 & 0.215 \\
\hline VIF (Max) & 4.57 & 4.57 & 4.36 & 4.41 \\
\hline VIF (Average) & 2.39 & 2.41 & 2.25 & 2.26 \\
\hline Observaciones & 867 & 871 & 821 & 821 \\
\hline
\end{tabular}

Nota: "Sî" o "No" indica si el sector industrial o el año de estudio son significativos a un nivel del 0.1 o niveles mayores o no. Las variables están definidas en la tabla 2. Los números reportados en paréntesis adoptan el estimador t. ${ }^{* * *},{ }^{* *}, *$ indican un nivel de significancia al $1 \%, 5 \%$ y $10 \%$, respectivamente.

En la tabla 7 se muestra el análisis de la relación entre las variables de género y composición del consejo y el nivel de endeudamiento. Se controlan los efectos fijos de sector industrial y año de estudio con variables dicotómicas. En los modelos 1 y 2 se incluyen como variables explicativas de diversidad de género en el consejo y equipo directivo, mientras que en los modelos 3 y 4 se integran las variables de composición del consejo como son su tamaño, independencia y dualidad COB-CEO. El modelo 1 evidencia que cuando la posición de $\mathrm{COB}$ es ocupada por una mujer incide negativamente en el nivel de endeudamiento total $(\mathrm{p}=0.01)$, mientras que el $\%$ de mujeres en el consejo no tiene incidencia. Empresas más rentables disminuyen el nivel de endeudamiento $(\mathrm{p}=0.01)$, mientras que los sectores de tecnologías de información, construcción servicios y bienes de consumo no básico, servicios de telecomunicaciones y salud, obtienen los mayores ratios de endeudamiento. El modelo 2 pone de manifiesto que la presencia de la mujer en el 
equipo directivo no tiene influencia significativa en el nivel de endeudamiento, mientras que el ROA lo disminuye ( $\mathrm{p}=0.01$ ). El modelo 3 confirma que las mujeres CEOs y que forman parte del equipo directivo disminuyen el nivel de endeudamiento $(\mathrm{p}=0.01 \mathrm{y} \mathrm{p}=0.10)$, mientras que el ROA lo disminuye. La dualidad COB-CEO incrementa el endeudamiento $(\mathrm{p}=0.01)$ mientras que la independencia del consejo lo disminuye $(\mathrm{p}=0.10)$. El modelo 4 demuestra que las mujeres CEOs no inciden en el endeudamiento, mientras que el \% de mujeres en el consejo lo disminuyen $(\mathrm{p}=0.05)$. El ROA y la dualidad COB-CEO reducen el nivel de endeudamiento y la independencia del consejo lo incrementan. Los resultados obtenidos permiten inferir que las mujeres que ocupan la presidencia del consejo reducen significativamente el endeudamiento $(\mathrm{p}=0.01)$, mientras que las mujeres CEO no tienen una influencia significativa.

La tabla 7, muestra el análisis de regresión múltiple utilizando el método de HuberWhite con errores estándar corregidos. Las variables dependientes se refieren a: (1) Endeudamiento total (TLEV) en el año t. Las variables independientes se centran en la diversidad de género en el consejo de administración (modelos 1 y 3 ) y la presencia de mujeres en el equipo directivo (modelos 2 y 4). Las variables de control incluidas en el modelo son el ROA, Tamaño de la Empresa, Edad de la Empresa, Tamaño del Consejo, Independencia del Consejo y Dualidad COB-CEO. Además, se incluyeron variables dummies para controlar por sector industrial y año de estudio.

Tabla 7. Regresión diversidad de género y nivel de endeudamiento total

\begin{tabular}{|c|c|c|c|c|}
\hline & (1) & $(2)$ & (3) & (4) \\
\hline Variables & TLEV & TLEV & TLEV & TLEV \\
\hline Mujer COB & $\begin{array}{c}-0.282^{* * *} \\
(-2.99)\end{array}$ & & $\frac{-0.233^{* * *}}{(-2.58)}$ & \\
\hline$\%$ mujeres consejo & $\begin{array}{l}-0.115 \\
(-1.57)\end{array}$ & & $\begin{array}{c}-0.154^{*} \\
(-1.86)\end{array}$ & \\
\hline Mujer CEO & & $\begin{array}{c}-0.01 \\
(-0.09)\end{array}$ & & $\begin{array}{l}0.065 \\
-0.52\end{array}$ \\
\hline$\%$ de mujeres equipo directivo & & $\begin{array}{l}-0.172 \\
(-1.27)\end{array}$ & & $\begin{array}{c}-0.314^{* *} \\
(-2.03)\end{array}$ \\
\hline ROA & $\begin{array}{c}-0.009^{* * *} \\
(-2.94)\end{array}$ & $\begin{array}{c}-0.009^{* * *} \\
(-2.95)\end{array}$ & $\begin{array}{c}-0.009^{* * *} \\
(-2.92)\end{array}$ & $\begin{array}{c}-0.009^{* * *} \\
(-2.93)\end{array}$ \\
\hline Tamaño de la empresa & $\begin{array}{c}0 \\
-0.05\end{array}$ & $\begin{array}{l}-0.001 \\
(-0.17)\end{array}$ & $\begin{array}{l}-0.004 \\
(-0.53)\end{array}$ & $\begin{array}{l}-0.005 \\
(-0.78)\end{array}$ \\
\hline Edad de la empresa & $\begin{array}{l}0.001 \\
-1.57\end{array}$ & $\begin{array}{l}0.001 \\
-1.42\end{array}$ & $\begin{array}{l}0.001 \\
-1.43\end{array}$ & $\begin{array}{l}0.001 \\
-1.15\end{array}$ \\
\hline Tamaño del consejo & & & $\begin{array}{c}0.003 \\
-1.1\end{array}$ & $\begin{array}{l}0.003 \\
-1.02\end{array}$ \\
\hline Independencia del consejo & & & $\begin{array}{c}-0.117^{*} \\
-1.69\end{array}$ & $\begin{array}{c}-0.117^{*} \\
(-1.70)\end{array}$ \\
\hline Dualidad COB-CEO & & & $\begin{array}{c}0.098^{* * *} \\
-4.52\end{array}$ & $\begin{array}{c}0.102^{* * *} \\
-4.72\end{array}$ \\
\hline Sector industrial & Sí & Sí & Sí & Sí \\
\hline Año de estudio & No & No & No & No \\
\hline
\end{tabular}


646 REMEF (The Mexican Journal of Economics and Finance)

Diversidad de género en posiciones estratégicas y el nivel de endeudamiento: Evidencia en empresas cotizadas mexicanas

\begin{tabular}{lcccc}
\hline & $(1)$ & $(2)$ & $(3)$ & $(4)$ \\
\hline \multicolumn{1}{c}{ Variables } & TLEV & TLEV & TLEV & TLEV \\
\hline Constante & $0.485^{* * *}$ & $0.506^{* * *}$ & $0.505^{* * *}$ & $0.533^{* * *}$ \\
& -4.54 & -4.74 & -4.58 & -4.89 \\
$R^{2}$ ajustado & 0.216 & 0.214 & 0.234 & 0.234 \\
VIF (Max) & 4.57 & 4.57 & 4.41 & 4.41 \\
VIF (Average) & 2.39 & 2.41 & 2.25 & 2.26 \\
Observaciones & 867 & 871 & 821 & 821
\end{tabular}

Fuente: elaboración propia con datos de los informes anuales de las empresas cotizadas. Nota: "Sí" o "No" indica si el sector industrial o el año de estudio son significativos a un nivel del 0.1 o niveles mayores o no. Las variables se encuentran definidas en la tabla 2. Los números reportados en paréntesis adoptan el estimador t. ${ }^{* *}, * *, *$ indican un nivel de significancia al $1 \%, 5 \%$ y $10 \%$, respectivamente.

\subsubsection{Diversidad de género y rentabilidad en empresas cotizadas mexicanas}

En esta sección se realizan los análisis econométricos para identificar si la diversidad de género en el consejo y equipo directivo inciden en la rentabilidad de la empresa (ROA). La tabla 8 evidencia que ninguna variable de género incide significativamente en la rentabilidad (ROA) de las empresas analizadas. Se observa que empresas de mayor tamaño obtienen ratios de rentabilidad más altos $(\mathrm{p}=0.01)$, mientras que el tamaño del consejo tiene una influencia significativa y positiva en la rentabilidad empresarial $(\mathrm{p}=0.10)$. Los resultados anteriores sustentan la premisa de la teoría de la masa crítica, la cuale stablece que una escasa representación de mujeres en posiciones de liderazgo no impactan en las decisiones del grupo mayoritario, y que es necesario incrementar su representación para obtener resultados significativos (Konrad et al., 2008; Torchia et al., 2011).

Tabla 8. Regresión diversidad de género y rentabilidad

Esta tabla presenta el análisis de regresión múltiple utilizando el método de Huber-White con errores estándar corregidos. La variable dependiente se refiere a la rentabilidad (ROA) en el año t. Las variables independientes se refieren a la diversidad de género en el Consejo de Administración (modelos 1 y 3 ) y la presencia de mujeres en el equipo directivo (modelos 2 y 4). Las variables de control incluidas en el modelo son el Endeudamiento a Largo Plazo, Tamaño de la Empresa, Edad de la Empresa, Tamaño del Consejo, Independencia del Consejo y Dualidad COB-CEO. Además, se incluyeron variables dummies para controlar por sector industrial y año de estudio.

\begin{tabular}{lcccc}
\hline & $(1)$ & $(2)$ & $(3)$ & $(4)$ \\
\hline \multicolumn{1}{c}{ Variables } & ROA & ROA & ROA & ROA \\
\hline Mujer COB & 0.588 & & 0.318 & \\
\% mujeres consejo & -0.31 & & -0.16 & \\
& -5.26 & & -5.035 & \\
Mujer CEO & $(-1.16)$ & & $(-1.19)$ & \\
& & -1.68 & & 0.033 \\
& & $(-0.65)$ & & -0.01
\end{tabular}


Revista Mexicana de Economía y Finanzas, Vol. 13 No. 4, (2018), pp. 631-654 647

DOI: http://dx.doi.org/10.21919/remef.v13i4.343

\begin{tabular}{|c|c|c|c|c|}
\hline & (1) & $(2)$ & $(3)$ & $(4)$ \\
\hline Variables & ROA & ROA & ROA & ROA \\
\hline $\begin{array}{l}\% \text { de mujeres equipo } \\
\text { directivo }\end{array}$ & & -12.876 & & -16.43 \\
\hline & & $(-1.17)$ & & $(-1.23)$ \\
\hline Endeudamiento a largo plazo & $\begin{array}{l}-6.349 \\
(-1.26)\end{array}$ & $\begin{array}{l}-6.511 \\
(-1.30)\end{array}$ & $\begin{array}{l}-7.914 \\
(-1.37)\end{array}$ & $\begin{array}{l}-7.965 \\
(-1.39)\end{array}$ \\
\hline Tamaño de la empresa & $\begin{array}{c}1.131^{* * *} \\
-4.67\end{array}$ & $\begin{array}{c}1.058^{* * *} \\
-4.45\end{array}$ & $\begin{array}{c}1.186^{* * *} \\
-4.55\end{array}$ & $\begin{array}{c}1.114^{* * *} \\
-4.47\end{array}$ \\
\hline Edad de la empresa & $\begin{array}{c}0.009 \\
-0.3\end{array}$ & $\begin{array}{l}0.003 \\
-0.08\end{array}$ & $\begin{array}{c}0.01 \\
-0.35\end{array}$ & $\begin{array}{l}0.003 \\
-0.09\end{array}$ \\
\hline Tamaño del consejo & & & $\begin{array}{c}0.226^{*} \\
-1.81\end{array}$ & $\begin{array}{c}0.212^{*} \\
-1.66\end{array}$ \\
\hline Independencia del consejo & & & $\begin{array}{l}0.346 \\
(0,09)\end{array}$ & $\begin{array}{l}0.367 \\
-0.09\end{array}$ \\
\hline Dualidad COB-CEO & & & $\begin{array}{l}-0.702 \\
(-0.47)\end{array}$ & $\begin{array}{c}-0.54 \\
(-0.36)\end{array}$ \\
\hline Sector industrial & Sí & Sí & Sí & Sí \\
\hline Año de estudio & Sí & Sí & Sí & Sí \\
\hline Constante & $\begin{array}{c}-9.03^{* *} \\
(-2.13)\end{array}$ & $\begin{array}{r}-7.823^{*} \\
(-1.87)\end{array}$ & $\begin{array}{c}-12.631^{* * *} \\
(-2.50)\end{array}$ & $\begin{array}{c}-11.305^{* * *} \\
(-2.40)\end{array}$ \\
\hline R2 ajustado & 0.064 & 0.066 & 0.08 & 0.215 \\
\hline VIF (Max) & 4.56 & 4.47 & 4.41 & 4.41 \\
\hline VIF (Average) & 2.4 & 2.42 & 2.27 & 2.28 \\
\hline Observaciones & 867 & 871 & 821 & 821 \\
\hline
\end{tabular}

Fuente: elaboración propia con datos de los informes anuales de las empresas cotizadas. Nota: "Sî" o "No" indica si el sector industrial o el año de estudio son significativos. Las variables están definidas en la tabla 2. Los números reportados en paréntesis adoptan el estimador $\mathrm{t}^{* * *}, * *, *$ indican un nivel de significancia al $1 \%, 5 \%$ y $10 \%$, respectivamente.

\subsection{Análisis Adicionales (Regresión Logit)}

En este apartado se adopta el análisis Logit para confirmar los resultados obtenidos en las tablas 6 y 7 . El análisis de la tabla 9 describe la relación entre la diversidad de género y el nivel de endeudamiento a largo plazo. Para ello se clasificó a las empresas en una variable dicotómica: aquellas empresas más endeudadas (valor de 1) o menos endeudadas (valor de 0 ) tomando como referencia la mediana del ratio de endeudamiento en cada año de estudio. Los resultados confirman que cuando las mujeres ocupan el cargo de COB, inhiben el nivel de endeudamiento a largo plazo $(\mathrm{p}=001)$, mientras que como consejeras no muestran una asociación significativa. Del mismo modo, cuando la posición de CEO es sostenida por una mujer, tiene un impacto negativo en el endeudamiento a largo plazo $(\mathrm{p}=0.05)$. Con respecto a las variables de control, se observa que el tamaño de la empresa favorece el nivel de endeudamiento a largo plazo $(\mathrm{p}=0.01)$, al igual que el tamaño del consejo $(\mathrm{p}=0.01)$ y la dualidad COB-CEO $(\mathrm{p}=0.01)$. Los resultados obtenidos nos sugieren qué en el caso de México, la mujer muestra una aversión al riesgo, particularmente en el caso del nivel de endeudamiento, aun cuando su participación es muy limitada. 
648 REMEF (The Mexican Journal of Economics and Finance)

Diversidad de género en posiciones estratégicas y el nivel de endeudamiento: Evidencia en empresas cotizadas mexicanas

La tabla 9, muestra los resultados del análisis de regresión Logit, la variable dependiente se refiere al endeudamiento a largo plazo (LLEV) en el año t, calculada como una variable dicotómica que toma el valor de 1 si la empresa obtiene un ratio de endeudamiento a largo plazo por encima del valor de la mediana en el año t, y de 0 en caso contrario. Las variables independientes se refieren a la diversidad de género en el consejo de administración (modelos 1 y 3) y la presencia de mujeres en el equipo directivo (modelos 2 y 4). Las variables de control incluidas en el modelo son el ROA, Tamaño de la Empresa, Edad de la Empresa, Tamaño del Consejo, Independencia del Consejo y Dualidad COB-CEO. Además, se incluyeron variables dummies para controlar por sector industrial y año de estudio.

Tabla 9. Regresión Logit relación de la diversidad de género y el nivel de endeudamiento a largo plazo

\begin{tabular}{|c|c|c|c|c|}
\hline & (1) & $(2)$ & $(3)$ & $(4)$ \\
\hline Variables & LLEV & LLEV & LLEV & LLEV \\
\hline \multirow[t]{2}{*}{ Mujer COB } & $-1.783^{* * *}$ & & $-1.451^{* *}$ & \\
\hline & $(-2.63)$ & & $(-2.37)$ & \\
\hline \multirow[t]{2}{*}{$\%$ mujeres consejo } & 1.423 & & 1.206 & \\
\hline & -1.31 & & -1.07 & \\
\hline \multirow[t]{2}{*}{ Mujer CEO } & & $-1.261^{* *}$ & & -0.288 \\
\hline & & $(-2.30)$ & & $(-0.29)$ \\
\hline \multirow[t]{2}{*}{$\%$ de mujeres equipo directivo } & & 0.265 & & 0.255 \\
\hline & & -1.41 & & -1.28 \\
\hline \multirow[t]{2}{*}{$\mathrm{ROA}$} & -0.006 & -0.006 & -0.008 & -0.008 \\
\hline & $(-0.76)$ & $(-0.75)$ & $(-0.93)$ & $(-0.94)$ \\
\hline \multirow[t]{2}{*}{ Tamaño de la empresa } & $0.180 * * *$ & $0.192^{* * *}$ & $0.127^{* * *}$ & $0.130 * * *$ \\
\hline & -3.92 & -4.2 & -2.54 & -2.61 \\
\hline \multirow[t]{2}{*}{ Edad de la empresa } & -0.004 & -0.002 & -0.005 & $-0.004^{* *}$ \\
\hline & $(-0.95)$ & $(-0.59)$ & $(-1.21)$ & $(-0.87)$ \\
\hline \multirow[t]{2}{*}{ Tamaño del consejo } & & & $0.125^{* * *}$ & $0.126^{* * *}$ \\
\hline & & & -4.97 & -4.96 \\
\hline \multirow[t]{2}{*}{ Independencia del consejo } & & & 0.01 & -0.129 \\
\hline & & & -0.02 & $(-0.24)$ \\
\hline \multirow[t]{2}{*}{ Dualidad COB-CEO } & & & $0.911^{* * *}$ & $0.898 * * *$ \\
\hline & & & -5.46 & -5.67 \\
\hline Sector industrial & Sí & Sí & Sí & Sí \\
\hline Año de estudio & No & No & No & No \\
\hline Constante & $\begin{array}{c}-3.502^{* * *} \\
(-3.95)\end{array}$ & $\begin{array}{c}-3.669 * * * \\
(-4.16)\end{array}$ & $\begin{array}{c}-5.028 * * * \\
(-5.02)\end{array}$ & $\begin{array}{c}-5.008 * * * \\
(-5.07)\end{array}$ \\
\hline$R^{2}$ ajustado & 0.091 & 0.092 & 0.129 & 0.13 \\
\hline Observaciones & 867 & 871 & 821 & 821 \\
\hline
\end{tabular}

Fuente: elaboración propia con datos de los informes anuales de las empresas cotizadas. Nota: "Sí" o "No" indica si el sector industrial o el año de estudio son significativos a un nivel del 0.1 o niveles mayores o no. Las variables se encuentran definidas en la tabla 2. Los números reportados en paréntesis se refieren al estimador $\mathrm{Z} . * * *, * *, *$ indican un nivel de significancia al $1 \%, 5 \%$ y $10 \%$, respectivamente. 
La tabla 10 demuestra empíricamente que la composición del consejo es una dimensión que incide significativamente en el nivel de endeudamiento total en empresas cotizadas mexicanas. Particularmente se observa que cuando la posición de COB es ocupada por una mujer, el ratio de endeudamiento total disminuye $(\mathrm{p}=0.01)$. Del mismo modo, la independencia del consejo tiene un efecto negativo sobre el nivel de endeudamiento total $(\mathrm{p}=0.01)$, mientras que la dualidad COB-CEO lo incrementa $(\mathrm{p}=0.05)$. Se observa que el sector industrial tiene un impacto significativo en las decisiones de apalancamiento. Estos resultados confirman que la participación de la mujer en posiciones estratégicas tiene incidencia en el resultado empresarial y van en línea con los hallazgos de Francis, Hasan y Wu (2013), quienes evidencian que las mujeres que participan en posiciones directivas son más aversas al riesgo al tomar decisiones financieras.

Tabla 10. Modelo Logit, relación de la diversidad de género y el nivel de endeudamiento a total

Esta muestra los resultados del análisis de regresión Logit, la variable dependiente se refiere al Endeudamiento Total (TLEV) en el año t, calculada como una variable dicotómica que toma el valor de 1 si la empresa obtiene un ratio de endeudamiento a largo plazo por encima del valor de la mediana en el año t, y de 0 en caso contrario. Las variables independientes se refieren a la diversidad de género en el consejo de administración (modelos 1 y 3 ) y la presencia de mujeres en el equipo directivo (modelos 2 y 4). Las variables de control incluidas en el modelo son el ROA, Tamaño de la Empresa, Edad de la Empresa, Tamaño del Consejo, Independencia del Consejo y Dualidad COB-CEO. Además, se incluyeron variables dummies para controlar por sector industrial y año de estudio.

\begin{tabular}{|c|c|c|c|c|}
\hline & (1) & $(2)$ & (3) & (4) \\
\hline Variables & TLEV & TLEV & TLEV & TLEV \\
\hline Mujer COB & $\begin{array}{c}-0.256^{* * *} \\
(-2.99)\end{array}$ & & $\begin{array}{c}-0.203^{* * *} \\
(-2.58)\end{array}$ & \\
\hline$\%$ mujeres consejo & $\begin{array}{l}-0.696 \\
(-0.99)\end{array}$ & & $\begin{array}{l}-0.998 \\
(-1.22)\end{array}$ & \\
\hline Mujer CEO & & $\begin{array}{l}-0.42 \\
(-0.46)\end{array}$ & & $\begin{array}{l}-0.167 \\
(-0.16)\end{array}$ \\
\hline$\%$ de mujeres equipo directivo & & $\begin{array}{l}-0.163 \\
(-0.90)\end{array}$ & & $\begin{array}{l}-0.184 \\
(-0.92)\end{array}$ \\
\hline ROA & $\begin{array}{l}-0.038 \\
(-1.41)\end{array}$ & $\begin{array}{l}-0.039 \\
(-1.41)\end{array}$ & $\begin{array}{l}-0.037 \\
(-1.28)\end{array}$ & $\begin{array}{l}-0.037 \\
(-1.28)\end{array}$ \\
\hline Tamaño de la empresa & $\begin{array}{l}0.053 \\
-1.11\end{array}$ & $\begin{array}{l}0.058 \\
-1.21\end{array}$ & $\begin{array}{l}0.023 \\
-0.43\end{array}$ & $\begin{array}{c}0.02 \\
-0.36\end{array}$ \\
\hline Edad de la empresa & $\begin{array}{l}0.001 \\
-0.34\end{array}$ & $\begin{array}{c}0 \\
-0.11\end{array}$ & $\begin{array}{c}0.002 \\
-0.5\end{array}$ & $\begin{array}{l}0.001 \\
-0.24\end{array}$ \\
\hline Tamaño del consejo & & & $\begin{array}{l}0.013 \\
-0.54\end{array}$ & $\begin{array}{l}0.012 \\
-0.49\end{array}$ \\
\hline Independencia del consejo & & & $\begin{array}{c}-2.233^{* * *} \\
(-4.27)\end{array}$ & $\begin{array}{c}-2.197^{* * *} \\
(-4.23)\end{array}$ \\
\hline Dualidad COB-CEO & & & $\begin{array}{c}0.601^{* * *} \\
-3.53\end{array}$ & $\begin{array}{c}0.617^{* * *} \\
-3.58\end{array}$ \\
\hline Sector industrial & Sí & Sí & Sí & Sí \\
\hline Año de estudio & No & No & No & No \\
\hline Constante & $\begin{array}{c}-1.477^{*} \\
(-1.68)\end{array}$ & $\begin{array}{c}-1.556^{*} \\
(-1.76)\end{array}$ & $\begin{array}{l}-0.567 \\
(-0.58)\end{array}$ & $\begin{array}{l}-0.531 \\
(-0.53)\end{array}$ \\
\hline R2 ajustado & 0.095 & 0.096 & 0.121 & 0.121 \\
\hline Observaciones & 867 & 871 & 821 & 821 \\
\hline
\end{tabular}

Fuente: elaboración propia con datos de los informes anuales de las empresas cotizadas. 
650 REMEF (The Mexican Journal of Economics and Finance)

Diversidad de género en posiciones estratégicas y el nivel de endeudamiento: Evidencia en empresas

cotizadas mexicanas

Nota: "Sî" o "No" indica si el sector industrial o el año de estudio son significativos a un nivel del 0.1 o niveles mayores o no. Las variables se encuentran definidas en la tabla 2. Los números reportados en paréntesis se refieren al estimador $\mathrm{Z}$. ${ }^{* * *}, * *, *$ indican un nivel de significancia al $1 \%, 5 \%$ y $10 \%$, respectivamente.

\section{Conclusiones, limitaciones y futuras líneas de investigación}

En la última década la relación entre la diversidad de género y el desempeño financiero ha recibido gran atención por parte de la academia y las instituciones a nivel mundial. Muestra de ello, son las cuotas de género impuestas en algunos países como Noruega y Australia. Sin embargo, en el contexto mexicano, la imposición de cuotas de género se ha limitado para el sector público y cargos de representación popular y no existe una regulación o prácticas de adopción voluntaria que motiven a las empresas a incrementar la diversidad de género en posiciones estratégicas. De ahí la importancia de este estudio, que demostró empíricamente la influencia que tiene la mujer en el desempeño financiero de empresas cotizadas, en un contexto caracterizado por una escasa representación femenina en puestos con mayor responsabilidad y un entorno institucional débil en materia de gobierno corporativo. Recientemente en México opera la ley 50-50 de equidad de género para ocupar cargos en el sector público, por lo que se recomienda promover medidas que fortalezcan la diversidad de género en el ámbito empresarial. Este trabajo extiende la literatura internacional en gobierno corporativo y diversidad de género en México, con el objetivo de sensibilizar y hacer un llamado a los tomadores de decisiones e instituciones reguladoras en el sector empresarial, para que a través de políticas o prácticas de gobierno corporativo la diversidad de género en el consejo y equipo directivo se incremente.

Al mejor de nuestro conocimiento, este estudio es uno de los pioneros en México, en demostrar empíricamente la influencia que tiene la mujer en posiciones del consejo y equipo directivo sobre el nivel de endeudamiento y la rentabilidad empresarial. Después de controlar por el tamaño e independencia del consejo, la dualidad COB-CEO y las características de la empresa como son su tamaño, edad, rentabilidad, sector industrial y año de estudio, se observó que la diversidad de género en el consejo disminuye el nivel de endeudamiento a largo plazo y total. Particularmente, los resultados empíricos a través de un análisis de datos de panel con efectos fijos y de una regresión Logit, sustentan que la posición de COB ocupada por una mujer incide negativa y significativamente en el nivel de endeudamiento a largo plazo y total, mientras que la posición de CEO ocupada por el género femenino tiene incidencia solamente en el nivel de endeudamiento a largo plazo. En este contexto, la hipótesis $H 1_{B}$ es aceptada, mientras que las $H 1_{A}$ es rechazada. Con respecto a la variable rentabilidad, se rechazan las hipótesis $H 2_{A} \mathrm{y}$ $H 2_{B}$, lo que nos sugiere que el desempeño financiero solamente se incrementa cuando la representación de las mujeres en los consejos tiene un nivel mayor al $30 \%$, mientras que a niveles bajos de participación, el desempeño podría verse reducido o no impactado (Joecks, Pull Vetter, 2013). A pesar de que la literatura previa no es concluyente, los resultados en el contexto mexicano nos sugieren que las mujeres que participan en posiciones estratégicas de empresas cotizadas, a pesar de su escasa representación, adoptan una actitud hacia la aversión al riesgo y una toma de decisiones cautelosa para incrementar el endeudamiento de la empresa (García-Meca, 2016).

Con respecto a la composición del consejo, se evidencia que esta variable está significativamente asociada a las decisiones de endeudamiento. Los resultados demuestran que el tamaño del consejo y la dualidad COB-CEO incrementan el nivel de endeudamiento a largo plazo y total, mientras que la independencia del consejo lo disminuyen. Los resultados obtenidos ponen de manifiesto que la limitada participación femenina en las 
empresas cotizadas mexicanas, no sustentan la premisa de la teoría de agencia, que afirma que la independencia del consejo motiva a un mayor endeudamiento y desempeño financiero, sin embargo, esto podría deberse a dos factores: 1) que las mujeres que participan en el consejo no son independientes y forman parte de la familia fundadora de la empresa, y 2) su limitada representación no incide significativamente en las decisiones corporativas, ya que de acuerdo con la teoría de la masa crítica, se requieren al menos tres mujeres en el consejo para que su impacto sea significativo en el resultado empresarial.

Los resultados obtenidos sugieren que es necesario que el sector empresarial adopte principios y políticas que promuevan el empoderamiento de la mujer y que visualicen una estrategia integral para lograr el crecimiento y desarrollo con un enfoque inclusivo con perspectiva de género. ONU Mujeres de México ha propuesto una serie de principios que motivan la igualdad de oportunidades, como el garantizar una participación creciente de mujeres $(30 \%)$, ofrecer condiciones laborales flexibles, invertir en políticas y programas de actualización en el lugar de trabajo, difundir las políticas empresariales y los planes de implementación a favor de la igualdad de género e integrar indicadores de género en las obligaciones de rendición de cuentas (ONU Mujeres, 2016). Es importante reconocer las limitaciones de este estudio. En primer lugar, la muestra de estudio está centrada en aquellas empresas que cotizan en la Bolsa Mexicana de Valores durante el periodo 20042016, y excluye a las empresas que pertenecen al sector financiero y bancario. Además, el estudio se centra en algunas variables financieras como el endeudamiento a largo plazo y total y la rentabilidad (ROA), y no se analizan otras variables financieras de riesgo o liquidez. Con estas limitaciones en mente, se abren nuevas líneas de investigación en el contexto mexicano, por ejemplo, extender la muestra a otros países latinoamericanos para realizar un estudio comparativo, así como incluir otras variables financieras de riesgo y rentabilidad. Se puede analizar también otras características de las mujeres que participan en posiciones de liderazgo como son su educación, lazos familiares con los fundadores, edad, experiencia, entre otras.

\section{Referencias}

Adams, R., Ferreira, D. (2009). Women in the boardroom and their impact on governance and performance. Journal of Financial Economics, 94(2), 291-309.

Ali, M., Ng, Y.L., Kulik, C.T. (2014). Board age and gender diversity: A test of competing linear and curvilinear predictions. Journal of Business Ethics, 125(3), 497-512.

Apesteguia, J., Azmat, G., Iriberri, N. (2012). The impact of gender composition on team performance and decision-making: Evidence from the field. Management Science, 58, 78-93.

Australian Securities Exchange (2010). Corporate Governance Principles and Recommendations with 2010 amendments. Available at http://www. asxgroup.com.au/media/PDFs/cg_principles_recommendations_ with_2010_amendments.pdf. Accessed 16 November 2012.

Bolsa Mexicana de Valores (2017). Empresas Listadas. Obtenido de: http://www.bmv.com.mx/es/empresaslistadas.

Briano-Turrent, G. (2014). Factores que inciden en una mayor transparencia de gobernanza corporativa en empresas cotizadas latinoamericanas. Revista Mexicana de Economía y Finanzas, 9(2), 105-124. 
652 REMEF (The Mexican Journal of Economics and Finance)

Diversidad de género en posiciones estratégicas y el nivel de endeudamiento: Evidencia en empresas cotizadas mexicanas

Bruckmüller, S. Branscombe, N.R. (2010). The glass cliff: When and why women are selected as leaders in crisis contexts. British Journal of Social Psychology, 49, 433-451.

Camarena, M., Saavedra, M. Aguilar, M. (2016). La relación entre la rentabilidad y la participación de las mujeres en las empresas que cotizan en la BMV. XXI Congreso Internacional de Contaduría, Administración e Informática, Ciudad de México, FCA-UNAM.

Camarena, M. Saavedra, M. (2018). El techo de Cristal en México. Revista de estudios de género la Ventana, 47, 312-347.

Campbell, K., Minguez- Vera, A. (2008). Gender diversity in the boardroom and firm financial performance. Journal of Business Ethics, 83(3), 435-451

CEPAL (2017). Mujeres en puestos de alta dirección en grandes empresas de América Latina. 27 de febrero de 2015. https://www.cepal.org/es/infografias/mujeres-en-puestos-de-alta-direccion-en-grandesempresas-de-america-latina (último acceso: 8 de diciembre de 2017).

Chapple, L., Humphrey, J. (2014). Does board gender diversity have a financial impact? Evidence using stock portfolio performance. Journal of Business Ethics, 122, 709-723.

Charness, G., Gneezy, U. (2012). Strong evidence for gender differences in risk taking. Journal of Economic Behavior Organization, 83, 50-58.

Coleman, S., Robb A. (2009). A comparison of new firm financing by gender: evidence from the Kauffman Firm Survey data. Small Business Economics, 33, 397-411.

Costa, P., Terracciano, A., McCrae, R. (2001). Gender differences in personality traits across cultures: Robust and surprising findings. Journal of Personality and Social Psychology, 81, 322-331.

Croson, R., Gneezy, U. (2009). Gender differences in preferences. Journal of Economic Literature, 47, $1-27$.

Crutchley, C.E., Hansen, R.S. (1989). A test of the agency theory of managerial ownership, corporate leverage, and corporate dividends. Financial Management, 18(4), 36-46.

Credit Suisse (2017). Gender diversity and corporate performance. Agosto de 2012. https://www . calstrs . $\mathrm{com} / \mathrm{sites} / \mathrm{main} / \mathrm{files} / \mathrm{file}$-attachments/csri_gender_diversity_and_corporate_performance. pdf (últimoacceso:8dediciembrede2017).

Eckel, C., Grossman, P. (2002). Sex differences and statistical stereotyping in attitudes toward financial risk. Evolution and Human Behavior, 23, 281-295.

El Economista (2017). Desigualdad laboral. Mujeres, con apenas $36.6 \%$ del empleo formal en México. Enero de 2017. Obtenido de: https://www.eleconomista.com.mx/empresas/Mujeres-con-apenas36.6-del-empleo-formal-en-Mexico-20170119-0020.html.

Esteban, L. (2007). Consejos de Administración y creación de valor: la perspectiva de género. Revista Española de Financiación y Contabilidad. XXXVI (135) 645-648.

Faccio, M., Marchica, M.T., Mura, R. (2016). CEO gender, corporate risk-taking, and the efficiency of capital allocation. Journal of Corporate Finance, 39, 193-209.

Francis, B., Hasan, I., Park, J.C., Wu, Q. (2014). Gender differences in financial reporting decisionmaking: Evidence from accounting conservatism. Contemporary Accounting Research, 32(3), 12851318.

Francis, B., Hasan, I., Wu, Q. (2012). The impact of CFO Gender on bank loan contracting. Journal of Accounting, Auditing Finance, 29(1), 53-78.

Ganga-Contreras, F.A., Ramos-Hidalgo, M.E., Leal-Millán, A.G., Valdivieso-Fernández, P.E. (2015). Teoría de agencia (TA): supuestos teóricos aplicables a la gestión universitaria. Innovar: Revista de ciencias administrativas y sociales, 25(57), 11-26.

González, C.F. (2016). De la representacion descriptiva a la representacion sustantiva. Analisis de las cuotas de genero en los congresos estatales en Mexico. Centro de Investigación y Docencia Económicas, A.C., CIDE.

Gordini, N., Racanti, E. (2017). Gender diversity in the Italian boardroom and firm financial performance. Management Research Review, 14(1), 75-94.

Graham, M.E., Belliveau, M.A., Hotchkiss, J.L. (2017). The view at the top of signing at the bottom? Work place diversity responsibility and women's representation in managment. ILR Review, 70(1), 223-258.

Gul, F. A., Srinidhi, B. y Ng, A.C. (2011). Does board gender diversity improve the informativeness of Stock Prices? Journal of Accounting and Economics, 51(3), pp. 314-338.

Huang, J., Kisgen, D.J. (2013). Gender and corporate finance: Are male executives overconfident relative to female executives? Journal of Financial Economics, 108, 822-839.

Huse, M. y Solberg, G. (2006). Gender-related boardroom dynamics: How Scandinavian women make and can make contributions on corporate boards. Women In Management Review, 21(2), pp. 113-130.

INEGI (2017). Indicadores de ocupación y empleo. Cifras oportunas durante diciembre de 2016. 20 de enero de 2017. 
Jeong, S.H., Harrison, D.A. (2017). Glass breaking, strategy making, and value creating: Meta-analytic outcomes of women CEO's and TMT members. Academy of Management Journal, 60, 1219-1252.

Joecks, J., Pull, K., Vetter, K. (2013). Gender diversity in the boardroom and firm performance: What exactly constitutes a Çritical Mass?". Academy of Management Journal, 60, 1219-1252.

Kanter, R. (1977). Some effects of proportions on group life: Skewed sex ratios and responses to token women. American Journal of Sociology, 82(5), 965-990.

Khaw, K.L.H., Liao, J., Tripe, D., Wongchoti, U. (2016). Gender diversity, state control, and corporate risk-taking: Evidence from China. Pacific-Basin Finance Journal, 39, 141-158.

Konrad, A.M., Kramer, V. (2006). 006). How many women do boards need? Harvard Business Review, $84(12), 22$.

Konrad, A.M., Kramer, V.W., Erkut, S. (2008). Critical mass: The impact of three or more women on corporate boards. Organizational Dynamics, 37(2), 145-164.

Liu, Y., Wei, Z., Xie, F. (2009). CFO gender and earnings management: evidence from China. Review of Quantitative Finance and Accounting, 46(4), 881-905.

Mateos de Cabo, R., Gimeno, R., Escot, L. (2010). Discriminación en consejos de administración: Análisis e implicaciones económicas. Revista de Economía Aplicada, 53(XVIII), 131-162.

McGuinness, P.B., Vieito, J.P., Wang, M. (2017). The role of board gender and foreign ownership in the CSR performance of Chinese listed firms. Journal of Corporate Finance, 42, 75-99.

McInerney-Lacombe, N., Bilimoria, D. y Salipante, P. (2008). Championing tough issues: How women corporate directors contribute to board deliberations. En S. Vinnicombe, V. Singh, R. J. Burke, D. Bilimoria y M. Huse, (Eds.). Women on corporate boards of directors: Research and practice, 123-139.

Miller, D., Le Breton-Miller, I., Lester, R. (2012). Family firm governance, strategic conformity, and performance: Institutional vs. strategic perspectives. The International Journal of Human Resource Management, 22(14), 2852-2873.

Mínguez-Vera, A., Martin, A. (2011). Gender and management on Spanish SMEs: an empirical analysis. The International Journal of Human Resource Management, 22(14), 2852-2873.

More, M. (2017). Por qué necesitamos a más mujeres en cargos directivos. 10 de mayo de 2017. http:// www.techexecutivesearch.es/por-que-necesitamos-a-mas-mujeres-en-cargos-directivos/ (último acceso: 28 de noviembre de 2017).

Nettle, D. (2007). Empathizing and systemizing: what are they, and what do they contribute to our understanding of psychological sex differences? British Journal of Psychology, 98, 237-255.

Nguyen, T., Locke, S., Reddy, K. (2015). Does boardroom gender diversity matter? Evidence from a transitional economy. International Review of Economics and Finance, 37, 184-202.

Palvia, A., Vähämaa, E., Vähämaa, S. (2015). Are female CEOs and chairwomen more conservative and risk averse? Evidence from the banking Industry during the financial crisis. Journal of Business Ethics, 131 (3), 577-594.

Pfeifer, C., Wagner, J. (2014). Age and gender effects of workforce composition on productivity and profits: Evidence from a new type of data for German enterprises. Contemporary Economics. 8(1), $25-45$.

Pindado, J., De la Torre, C. (2006). The role of financing, investment and dividend decisions in explaining corporate ownership structure: Evidence from Spain. European Financial Management, 12(5), 661-687.

Pucheta, M.C. Sánchez, P. (2013). Relación entre la diversidad de género y la rentabilidad económica de las empresas del IBEX 35. La ventana. Revista de Estudios de Género, 12(5), 661-687.

OCDE (2016). Conferencia sobre la mejora del acceso de mujeres a posiciones de liderazgo. Informe de fondo, 8 de marzo de 2016, París, p. 6.

ONU Mujeres (2016). Empresas y empoderamiento de las mujeres. Principios para el empoderamiento de las mujeres en las empresas. ONU Mujeres, México.

OIT (2017). La mujer en la gestión empresarial: Cobrando impulso en América Latina y el Caribe. Editado por Oficina de actividades para los empleadores. mayo de 2017. http://www.ilo.org/public/ spanish/dialogue/actemp/downloads/events/2017/lima_conf/wibm_fullreport_2017_sp.pdf (último acceso: 07 de Diciembre de 2017).

Rodríguez Nava, A., Venegas Martínez, F. (2012). Liquidez y apalancamiento de la banca comercial en México. Análisis Económico, XXVII(66), 73-96.

Srinidhi, B., Gui, F. A., Tsui, J. (2011). Female directors and earnings quality. Contemporary Accounting Research, 28(5), 1610-1644.

Terjesen, S., Barbosa Couto, E., Paulo Morais F. (2015). Does the presence of independent and female directors impact firm performance? A multi-country study of board diversity. Journal of Management Governance, 20(3), 447-483.

Torchia, M., Calabro, A., Huse, M. (2011). Women directors on corporate boards: From tokenism to critical mass. Journal of Business Ethics, 702(2), 299-317 
654 REMEF (The Mexican Journal of Economics and Finance)

Diversidad de género en posiciones estratégicas y el nivel de endeudamiento: Evidencia en empresas cotizadas mexicanas

Vithessonthi, C., Tongurai, J. (2015). The effect of leverage on performance: Domestically-oriented versus internationally-oriented firms. Research in International Business and Finance, 34, 265-280.

Zapata-Rotundo, G., Hernández-Arias, A. (2010). Sistema de incentivos y tipos básicos de trabajo en la organización bajo la perspectiva de la teoría de agencia. Pensamiento y Gestión, 29(Julio), 56-86. 